\title{
PERSPECTIVA DEL ECOTURISMO EN EL ALTIPLANO CUNDIBOYACENSE PARA CONFORMAR UNA RED LOCAL
}

\section{PERSPECTIVE OF ECOTOURISM IN THE CUNDIBOYACENSE HIGHLANDS TO FORM A LOCAL NETWORK}

\author{
Jairo Alberto Vásquez Bernal ${ }^{1}$, Adriana Posada Arrubla², Pedro Laytón Coy³
}

\begin{abstract}
${ }^{1}$ Mercadotecnista, Esp Gestión Social y Ambiental, Docente Investigador Facultad Ingeniería Comercial Universidad de Ciencias Aplicadas y Ambientales U.D.C.A. E-mail: jvasquez@udca.edu.co. ${ }^{2}$ Economista Agrícola, M.Sc. Planeación Urbana y Regional, Esp Gestión Social y Ambiental, decana Ingeniería Comercial U.D.C.A. E-mail: aposada@udca. edu.co. ${ }^{3}$ Ingeniero Comercial. Especialista en Finanzas, Docente Investigador Ingeniería Comercial U.D.C.A E-mail: playton@udca.edu.co. Dirección para correspondencia: calle 222 No 55 -37, Bogotá - Colombia.
\end{abstract}

Rev. U.D.C.A Act. E Div. Cient. 13 (1): 147-156, 2010

\section{RESUMEN}

El desarrollo del turismo a gran escala ha permitido la sub-clasificación del sector en categorías, tales como el ecoturismo. En Colombia tiene un importante potencial, especialmente, por la existencia de sus innumerables recursos naturales, los que representan atractivos ecoturísticos. Siendo el ecoturismo un concepto relativamente nuevo, con frecuencia las actividades desarrolladas no corresponden a lo normatizado o definido y, además, está asociado con otros términos, lo hizo necesario indagar y contrastar acerca de lo que realmente significa y la manera de implementarse en los municipios del altiplano Cundiboyacense, en Colombia. Este proceso incluyó la revisión bibliográfica, la identificación de la población, la selección de unidades objetivo del estudio, la comunidad, los turistas, los empresarios y las autoridades municipales y, la aplicación de un instrumento para la obtención de datos, del registro y posterior análisis de los mismos. Esto permitió, por una parte, establecer que los habitantes y los turistas no tienen claro lo que es ecoturismo, por lo cual, se llegó a una definición, cuyos aspectos fundamentales corresponden a lo que buscan los usuarios, las actividades que realizan, el propósito por la preservación de los atractivos naturales y los beneficios para las comunidades y, por otra, para la implementación, se estructuró un esquema de redes, entendiendo que las empresas, los turistas, la comunidad, el atractivo natural, las autoridades municipales y ambientales, se interrelacionan por medio de las actividades ecoturísticas, creando un sistema complejo de servicios, posibilitando a las organizaciones, que componen la red, el crecimiento y el desarrollo sostenible.

Palabras clave: Actividad ecoturística, atractivos naturales, turistas, comercio, esquema de redes.

\section{SUMMARY}

Tourism development has allowed large-scale subsector classification in categories such as ecotourism. This category has in Colombia a significant potential, especially, because of the existence of innumerable natural resources, representing eco-touristic attractions. Since ecotourism is a relatively new concept, its activities often do not correspond to normed or defined points of view, and is also associated with other terms; therefore it became necessary to investigate and contrast it means and how to be implemented in the municipalities of the 
altiplano Cundiboyacense in Colombia. This process included a literature review, the identification of the population, the selection of target and study units, community, tourists, businessmen and city officials; besides the application of an instrument for data collection, registration and subsequent data analysis was carried out. This allowed, initially, to establish that residents and tourists do not have a clear concept about what is ecotourism, which had as result the proposal of a definition, based on what potential users look for, the activities they accomplish, the purpose of preserving the natural attractions and the benefits for the involved communities. On the other hand, to implement these aspects, a network scheme was constructed, taking into account, that businesses, tourists, community, natural beauty, municipal and environmental authorities are interrelated through ecotourism activities, developing a complex system of services, enabling the organizations that comprise the network, a sustainable growth and development.

Key words: Ecotourism activity, natural attractions, turists, business, network.

\section{INTRODUCCIÓN}

El ecoturismo es el subsector del turismo que representa más dinámica económica (Puig, 2006) y profundas repercusiones sociales y políticas, por la distribución del acceso y del control sobre los recursos naturales (Kent, 2003), lo cual, se debe al interés por la conservación de los ecosistemas, la biodiversidad, el conocimiento de culturas y los beneficios que genera.

El ecoturismo es uno de esos conceptos que ha rondado por más de 20 años sin acuerdos generales; su uso, se inició en la década de los 80 y se emplean indistintamente, los términos turismo natural y ecoturismo. Según Pérez de las Heras (2003) es un concepto asociado con turismo rural, de campo, verde, de aventura, agroturismo, entre otros, especialmente, a nivel comercial, con el fin de atraer viajeros conscientes.

Por lo anterior, se indagaron varias fuentes y se encontraron diversas definiciones, desde ópticas diferentes, que no ayudan a la comprensión del término, sino que aportan aspectos puntuales que dificultan su entendimiento, como se establece en los siguientes significados.
Entre las primeras definiciones de ecoturismo, se destaca la elaborada por el arquitecto Ceballos (1991), indicando que es aquella modalidad turística ambientalmente responsable, que consiste en viajar y visitar áreas naturales, a través de un proceso que promueva la conservación, el bajo impacto ambiental y propicia un involucramiento activo y socioeconómico benéfico de las poblaciones locales. Institucionalmente, se destaca la de la Organización Mundial del Turismo OMT (2002), que definió ecoturismo como un fenómeno que engloba toda una serie de opciones, que varían desde un enfoque purista científico hasta la visita de recreo a una zona natural, como actividad de fin semana o como parte de un viaje más importante.

En el país existe la definición en la Ley 300 de 1996 (Colombia, 1996), la cual fue modificada por la Ley 1101 de 22 (Colombia, 2006); allí se establece que ecoturismo es el turismo especializado y dirigido, que se desarrolla en áreas con un atractivo natural especial y se enmarca dentro de los parámetros del desarrollo humano sostenible. Busca la recreación, el esparcimiento y la educación del visitante, a través de la observación, el estudio de los valores naturales y de los aspectos culturales relacionados.

En el siglo XXI, se destacan los conceptos de Lara de Vicente (2005), quien afirma que el ecoturismo se debe al interés por la conservación de ecosistemas, la biodiversidad, la flora, la fauna, el conocimiento de culturas; el de Rhodes (2005), quien establece que es una forma sustentable de turismo basado en recursos naturales y el del Anuario de Turismo y Sociedad (2005), que indica que es una forma de turismo que se desarrolla en áreas con un atractivo natural y se enmarca dentro de los parámetros de desarrollo humano sostenible. También es importante indicar que como actividad originada a partir de la existencia de un atractivo natural y con una dinámica propiciada por la participación del turista, genera un impacto global, no solo en el ambiente sino en la economía y en la sociedad (Cámara et al. 2005).

De todas las definiciones existentes, se resaltaron los elementos que ayudan a comprender mejor el ecoturismo y permitieron concretar el marco de trabajo en el contexto del altiplano Cundiboyacense, subregión colombiana de tierras altas y planas, localizadas en la cordillera oriental de los Andes colombianos, agrupada en tres regiones: la Sabana de Bogotá, los valles de Ubaté y Chiquinquirá y los de Duitama y Sogamoso. 
Esta región está en la jurisdicción de dos Corporaciones Autónomas Regionales, la CAR y CORPOBOYACA (I .A. v. H., 2004). El altiplano Cundiboyacense es el área geográfica objeto de estudio, en la cual, se localizan los empresarios, los turistas y la comunidad, que requieren comprender el ecoturismo, con un enfoque que incluya lo político, lo ecológico y lo social, para poder establecer una red de turismo.

Es así, como la propuesta del concepto de ecoturismo se apoya en la lingüística, en la cual, se especifica que la definición es la explicación de la complejidad, a través del género que lo superordina y la diferencia específica que lo produce (Rivano, 2005), es decir, es la declaración de las propiedades entre un término y el significado de éste, que corresponden a tipos y técnicas existentes como: la lexicológica, entendida como la definición descriptiva, por que conduce al significado de las palabras, mediante la enumeración de sus características (Brenes E Porras, 2004); la intencional, por el listado de términos que proporcionan todas las propiedades requeridas del objeto, para caer dentro del campo de la palabra definida (Munárriz E Domínguez, 2006) y la estipulativa, como el tipo de definición en la que un término nuevo o bien ya preexistente se le da un nuevo significado (Haba, 2004).

Estas definiciones, entre otras, son las adecuadas para comprender el concepto de ecoturismo, en tanto que permiten la interpretación del término de una manera clara, con fines de aplicación de su significado en las redes ecoturísticas; sin embargo, no son los suficientemente específicas para los actores que participarían en la conformación de la red, por lo cual, se hace necesario redefinir ecoturismo, desde la perspectiva del estudio.

Según Fernández (2005), una red es un sistema coordinado de relaciones de intercambio establecido por empresas mientras configuran su entorno, a través de un sistema integrado, con interdependencias funcionales y una identidad característica; vinculada por medio de acuerdos de cooperación, donde el principio de la red es la autoorganización.

En ese sentido, se pronuncia Galán (2007), cuando define la estructura empresarial, como una red externa organizacional de relaciones entre unidades de trabajo autónomo o semi-autónomo, para entregar un producto o servicio a un cliente. Para abordar lo pertinente a redes de empresas ecoturísticas, Lara de Vicente (2005) define red de turismo sostenible, como la cooperación e intercambio de información entre actores, con el fin de convertirse en un medio que motive y sensibilice a la creación y el establecimiento de procesos y de acciones dirigidas hacia un desarrollo sostenible del turismo.

Según lo anterior, La Red de ecoturismo se convierte en un mecanismo facilitador de procesos para el intercambio de información y vinculación de actores interesados en el desarrollo de la actividad turística del país (Fred, 2008), lo cual, busca promover espacios de concertación y de reflexión sobre el ecoturismo en el país, impulsando la cultura de la conservación, a través de investigación e intercambio de experiencias.

\section{MATERIALES Y MÉTODOS}

El proceso de indagación sobre diversos conceptos, como el de ecoturismo para generar uno propio, exige posiciones críticas, lo que requiere de un estudio analítico. De igual manera, la determinación de relaciones entre actividades, procesos y sistemas, en este caso el ecoturismo y la sostenibilidad, requiere de una investigación aplicada y cuantitativa, que incluye la clasificación y cruce de variables, el registro, la descripción, el análisis, la interpretación de resultados y, por último, el proceso, desarrollado por la identificación de la población (habitantes, turistas y empresarios) y por la determinación del tamaño de la muestra para cada unidad objeto de estudio, por la distribución muestral de las proporciones. En el caso de los turistas el proceso fue el siguiente:

$$
\mathrm{n}=\frac{\mathrm{Z}^{2} \times P \times Q}{E^{2}}
$$

Se realizó una pre-encuesta de tres preguntas, de respuesta dicotómica, con la que se obtuvo que, $\mathrm{P}$ (probabilidad porcentual de éxito de un suceso) $=59 \%$; $\mathrm{Q}$ (probabilidad porcentual de fracaso de un suceso) $=$ $41 \%$; Z (intervalo de confianza) $=0,83$ y E (error) $=5 \%$, con lo que se determinó que la cantidad de unidades de objeto de estudio para turistas fue de 97.

Para la comunidad, el tamaño de muestra se desarrolló con el siguiente procedimiento:

$$
n=\frac{Z^{2} \times P \times Q \times N}{E^{2}(N-1)+Z^{2} \times P \times Q}
$$


Se aplicó la preencuesta de tres preguntas, de respuesta dicotómica, y de ella, se obtuvo que, $P=56,66 \%$; $Q$ $=43,33 \% ; Z=0,89 ; N$ (tamaño de la población) $=$ 88.828 y $E$ (error) $=4,5 \%$, dando como resultado que la cantidad de unidades de objeto de estudio para comunidad fue de 96. El tamaño de muestra para las empresas, se obtuvo con la fórmula anterior, cuyos datos son: $\mathrm{P}=70 \% ; \mathrm{Q}=30 \% ; \mathrm{Z}=1,04, \mathrm{~N}=155$ y $\mathrm{E}$ (error) $=5 \%$, dando como resultado que la población a estudiar fue de 91 .

La selección de las provincias y de los municipios del altiplano Cundiboyacense, se realizó por varios criterios, como la distancia de Bogotá, el número de habitantes, la temperatura, el tipo de turismo, la cantidad de atractivos, los eventos y las actividades ecoturísticas, definiendo para el presente estudio a Alto Ricaurte y Tundaza, en Boyacá y Almeidas y Ubaté, en Cundinamarca.

El proceso de muestreo utilizado fue el probabilístico estratificado proporcional por cada tipo de unidad objeto de estudio: comunidad, empresas y turistas, como se muestra en la tabla 1.

La información primaria, se obtuvo por medio de observación directa $Y$ entrevistas en un formato estructurado directo, donde se interrogaba a los informantes acerca del concepto de ecoturismo, desarrollo sostenible, el lugar de procedencia, los atractivos de los municipios, los servicios ofrecidos, el tiempo de estadía de los turistas, la frecuencia, la inversión realizada, los impactos y la intención de conformar una red ecoturística. Esta información recopilada, se tamizó, se tabuló y se analizó, de tal forma que se integraron los aspectos, los elementos y las características en una propuesta de aplicación del ecoturismo que permitió reunir las propiedades generales, coherentes y diferenciadores del concepto de ecoturismo, desde la óptica de la comunidad del altiplano. Finalmente, se especifica que la investigación fue propositiva, por que planteó el mejoramiento de las condiciones del subsector, a través de la estructuración de una red, como un sistema complejo conformado por empresas, comunidad, turistas y Estado, interrelacionándose con los atractivos de los municipios.

\section{RESULTADOS Y DISCUSIÓN}

Con la información recopilada y considerando que son los turistas, empresarios, comunidad y autoridades municipales de la región, los interesados en desarrollar ecoturismo y quienes necesitan comprender el concepto, se propone, de una parte, la estructuración del concepto basado en sus opiniones y, de otra, la propuesta para la conformación de la red.

Tabla 1. Proceso de muestreo realizado en el Altiplano Cundiboyacense.

\begin{tabular}{|c|c|c|c|c|}
\hline PROVINCIAS & MUNICIPIOS & COMUNIDAD & EMPRESAS & ECOTURISTAS \\
\hline Alto Ricaurte & $\begin{array}{c}\text { Moniquirá } \\
\text { Arcabuco } \\
\text { Ráquira } \\
\text { Sutamarchán } \\
\text { Villa de Leyva }\end{array}$ & 15 & 36 & 49 \\
\hline Tundama & $\begin{array}{c}\text { Paipa } \\
\text { Duitama } \\
\text { Sogamoso }\end{array}$ & 39 & 22 & 13 \\
\hline Ubaté & $\begin{array}{c}\text { Ubaté } \\
\text { Fúquene } \\
\text { Lenguazaque Simijica }\end{array}$ & 34 & 19 & 12 \\
\hline Almeidas & $\begin{array}{l}\text { Villa Pinzón } \\
\text { Suesca }\end{array}$ & 8 & 14 & 23 \\
\hline TOTAL & & 96 & 91 & 97 \\
\hline
\end{tabular}


De los resultados de la encuesta a los empresarios, el $80 \%$ define ecoturismo como el turismo relacionado con actividades realizadas en la naturaleza, el $13 \%$, como turismo ecológico, que permite preservar el medio ambiente y un $7 \%$ admite no conocer el concepto. Así mismo, el 32,58\% de la comunidad seleccionada de los municipios que conforman el altiplano Cundiboyacense indicó que no sabe el significado de ecoturismo, el resto expresa muchos términos asociados a la actividad.

Respecto a las respuestas proporcionadas por los turistas, se establece que una parte importante no tiene claro que es el ecoturismo, pero disfrutan de sus actividades, sus funciones, sus servicios y sus beneficios en los atractivos de los municipios; además, se ha vuelto una actividad interesante para muchas personas, pero aun es un subsector incipiente y potencial de la economía. Esta situación hizo necesario indagar en otras fuentes secundarias y, sobretodo, profundizar en el significado de ecoturismo desde otras disciplinas, como son la ecología y la economía: a nivel ecológico, se destaca la definición de la Unión Mundial para la Naturaleza, que indica que es aquella modalidad turística ambientalmente responsable, que consiste en viajar o visitar áreas naturales relativamente sin disturbar, con el fin de disfrutar, de apreciar y de estudiar los atractivos naturales de dichas áreas, así como cualquier manifestación cultural que pueda encontrarse ahí, a través de un proceso que promueve la conservación. Además, añade, que tiene bajo impacto ambiental y benéfico de las poblaciones locales (Centro de Estudios Agropecuarios, 2001).

Otra definición, con enfoque ecologista, es la del Instituto von Humboldt (2000), que sostiene que el ecoturismo es aquel turismo interesado en visitar espacios con valores naturales, entre los que se encuentran áreas protegidas y conocer la flora y la fauna de los destinos visitados.

Obviamente, desde el enfoque ecológico, hay una fuerte propensión por establecer que la actividad turística se desarrolla en o alrededor de los recursos naturales y que son éstos los elementos, nucleares del concepto. Por ello, se encuentran definiciones que utilizan otras denominaciones, como atractivos naturales, espacios con valores naturales, atractivo natural especial, valores naturales, aspectos culturales, naturaleza, áreas naturales relativamente poco alteradas o no contaminadas, atractivo natural especial, paisajístico y cultural, conservación del medio ambiente, áreas naturales, áreas relativamente vírgenes, medio, tanto natural como cultural y áreas naturales relativamente sin disturbar.

Por su parte, desde el enfoque de la economía y disciplinas afines, algunas de estas definiciones son: el Ecoturismo es aquel sector especializado del turismo que se caracteriza por la marcada propensión a viajar, para estar en contacto con la naturaleza mediante el disfrute de ella, por simple observación o por su estudio sistemático (Díaz, 2001). Adicionalmente, Palafox (2005) incluye la educación, la recreación, la admiración y la interpretación del medio natural, a través de la relación simbiótica entre el turismo, el medio natural y los servicios, con enlaces a los recursos naturales y la gente que habita en el lugar.

Para la economía y disciplinas afines, se enfatiza en la actividad que se ejerce, tal es el caso de la recreación, el esparcimiento y la educación del visitante, el viaje individual $y$, especialmente, la relación que se genera entre el atractivo natural, la comunidad y los servicios que se derivan de esta dinámica. También, se destaca el hecho de concebir al ecoturismo como un subsector, fenómeno o actividad especializada.

En Colombia, existen declaraciones institucionales sobre ecoturismo, como la de la Ley 300/96 (Colombia, 1996; 2002): Es el turismo especializado y dirigido que se desarrolla en áreas con un atractivo natural especial y se enmarca dentro de los parámetros del desarrollo humano sostenible. Busca la recreación, el esparcimiento y la educación del visitante, a través de la observación, el estudio de los valores naturales y de los aspectos culturales relacionados con ellos.

La definición del Ministerio de Medio Ambiente (Colombia, 2006), en su política para el desarrollo de ecoturismo, lo define como una de las actividades en las que se hace más viable la implantación de modelos de desarrollo sostenible, ofreciendo al visitante la posibilidad de disfrutar de la oferta ambiental de un área geográfica, representada ya sea en su diversidad biológica o ecosistémica y que benefician, en primera instancia, a las comunidades que viven en las zonas de influencia de las áreas protegidas o de otra con atractivos para los visitantes.

En estas definiciones, se señalan viajes responsables, lo que significa que estos deben causar el menor impacto 
al área visitada y para ello es necesario crear conciencia acerca de la conservación y la sostenibilidad de los atractivos naturales; adicionalmente, el ecoturismo solo se puede desarrollar en zonas con alto potencial natural, donde se presente la oportunidad para el visitante de admirar la flora y la fauna, generando un sentido de pertenencia. Estos aspectos, se evidencian en lo expresado por la comunidad, los turistas y los empresarios.

Aunque para la comunidad no es claro el concepto de ecoturismo, se conoce las actividades que se realizan y las relaciones que se presentan entre los diferentes actores que participan en la actividad ecoturística; identifican plenamente los recursos naturales existentes y los posibles impactos y los beneficios que se derivan de la misma, es decir, comprenden el proceso general. Falta que establezcan los objetivos, las estrategias y las acciones comerciales que potencialicen el ecoturismo en la región, como sub-sector económico generador de recursos para la sociedad y para el sostenimiento y preservación del atractivo natural.

Se destacan diferentes aspectos para la definición de ecoturismo y se fundamenta, especialmente, en los criterios expuestos por los turistas, los empresarios y la comunidad; así mismo, en los antecedentes establecidos en la revisión documental. Un primer aspecto es la percepción holística del concepto y cómo lo identifican, indicando que es turismo especializado, turismo de naturaleza, servicio especializado y modalidad de turismo; otro aspecto es el lugar donde se desarrolla la actividad y, específicamente, la denominan como un atractivo natural.

Otros aspectos que se tienen en cuenta en la definición propuesta de ecoturismo son los expuestos por los turistas y lo que buscan en la implementación de la actividad, como la recreación, el esparcimiento, la educación, la cultura, la investigación y el deporte. El fin específico es conservar la naturaleza siendo responsable con el ambiente, garantizar la sostenibilidad del atractivo y contribuir al mantenimiento del patrimonio cultural de la región.

Finalmente, coinciden los turistas en qué actividades están relacionadas con el ecoturismo, como naturalismo, senderismo, ciclo-montañismo, surfing, esquí acuático, turismo ecológico, agroturismo, de aventura y ambiental, y que del ofrecimiento de estos se beneficia la comunidad, por la generación de empleo, contribuyendo al impulso económico por la participación a la economía y a la generación de ingresos.

Por lo anterior, se determinó que las diversas definiciones de ecoturismo puede originar errores conceptuales, las cuales, hacen que las estrategias socio-económicas municipales no sirvan como herramientas útiles para el fomento del ecoturismo y el desarrollo sostenible. Igualmente, es importante tener en cuenta, como lo señala Lagunas (2008), que administrar pensando en el ambiente requiere comprender cómo se vinculan el comercio, la competitividad y los recursos globales.

Con base en esta percepción y buscando que los actores que intervienen en una red de servicios ecoturisticos comprendan y se identifiquen con el concepto, se estable que el ecoturismo es una actividad del sector turístico en áreas, con atractivos naturales, en donde se realizan actividades específicas, que buscan la conservación y la interrelación de los turistas con los recursos naturales, el beneficio social y económico de la comunidad.

En Colombia, la actividad ecoturística se viene desarrollando lentamente y, en algunos lugares, de manera más organizada que en otros, pero aun es un subsector potencial de la economía y, en este contexto, tal potencial hace necesario su implementación en las regiones y subregiones colombianas, con el fin de poder darle salida a una posibilidad de progreso, propia de las condiciones naturales y culturales.

Otro resultado de este trabajo es la propuesta para aplicar el concepto de ecoturismo, a través de la estructuración de la red de servicios ecoturísticos, estableciendo algunos parámetros para su conformación, que garanticen, de una parte, la sostenibilidad de los atractivos y, de otra, la rentabilidad de los servicios ofrecidos por cada unidad empresarial.

Respecto a los resultados relacionados con el mercado del ecoturismo, la comunidad del altiplano Cundiboyacense consideró que los atractivos de los municipios son el paisaje y el clima $(18,55 \%)$, los volcanes y nacimientos de agua $(17,74 \%)$, los santuarios de flora y fauna $(15,32$ $\%)$; también indicaron que las actividades que realizan son turismo de naturaleza $(28,43 \%)$, gastronómico $(28,43 \%)$ y de aventura (15,69\%). Finalmente, el $86,46 \%$ de la comunidad afirmó que participaría en programas de ecoturismo. 
Los empresarios expresaron que los servicios mas importantes ofrecidos en el altiplano son hospedaje $(38,46 \%)$, comercio $(25,27 \%)$ y alimentación $(20,88 \%)$ y que los impactos positivos del ecoturismo son el aumento en las ventas $(41,76 \%$ y el desarrollo socio-económico, (29,67\%); respecto a los impactos negativos, la inseguridad es la que sobresale con un $23,08 \%$. Trasciende en el estudio que un $95,6 \%$ de los encuestados participaría en la conformación de una red de ecoturismo. Ellos tienen claro el funcionamiento del ecoturismo, como actividad económica y el ofrecimiento de servicios que se derivan del mismo, pero las costumbres y la cultura regional limitan la posibilidad de conformar una red. De acuerdo a lo que informaron están en capacidad de atender en promedio por día a 26 personas y el ingreso que genera cada uno de ellos es de $\$ 33.516$, lo cual, muestra que es un mercado potencialmente clave en la economía de los municipios.

Los turistas que frecuentan el altiplano Cundiboyacense, lo visitan por recreación y deporte $(25,77 \%)$ y para realizar actividades ecoturísticas (23,71\%). Los servicios más utilizados son alimentación $(23,71 \%)$ y hospedaje (15,46\%); también expresan que no tienen una época fija para visitar los municipios $(58,76 \%)$ y que viajan en familia (52,58\%).

Los resultados permiten inferir, primero, que los turistas desarrollan actividades propias del ecoturismo y que utilizan servicios complementarios, que son los elementos vitales con los atractivos naturales para la conformación de una red de ecoturismo y, segundo, que el flujo de visitantes se presenta en grupos y periódicamente durante el año, evidenciando un escenario óptimo para establecer convergencias y unificación conceptual y de servicios alrededor del ecoturismo.

Ahora bien, los turistas resaltan la posibilidad de disfrutar un mayor número de atractivos naturales, diversidad cultural y mejores precios en el altiplano (96,9\%), si se compara con el eje cafetero, en donde prevalece la organización y la diversidad de servicios complementarios (12,5\%).

Para el establecimiento de redes, Sandoval (2007) llevó a cabo una propuesta de integración de servicios a las empresas operadoras realizando acciones responsables que propendan por el desarrollo sostenible. Por su parte, Morrison, citado por Andreu \& Parra (2005), propone un sistema de relaciones formales entre los elementos del servicio, generando al destino y a las empresas turísticas una capacidad competitiva y sostenible.

Según Lovelock E Wirtz (2009), poner en contacto personas cuyos intereses son similares, permiten el desarrollo de redes con clientes, con distribuidores, con proveedores, con medios masivos, con consultores, con asociaciones de comercio y con agencias gubernamentales. Muñoz (2005) expresa que cuando una persona adquiere un servicio, no lo elige de acuerdo a la versión del todo, sino que lo descompone en características o partes y comprueba si coinciden con las que va buscando. La relación existente entre las actividades complementarias que ofrezcan la red y el aprovechamiento del atractivo no es vista en conjunto por el turista, sino que la percepción de organización y la posibilidad de ir empleando los diferentes servicios, de manera coordinada, es lo que genera un grado mayor de satisfacción.

Ahora, la comprensión del ecoturismo desde la perspectiva del servicio implica entenderlo como un sistema complejo conformado por las actividades ecoturísticas y los servicios complementarios, integrada por la comunidad, los turistas, el Estado, interrelacionándose con los atractivos de los municipios, en donde cada participante, mantiene su autonomía e independencia, para la toma de decisiones.

Los atractivos naturales son el elemento funcional de la red, sobre los cuales, se desarrolla el ecoturismo y son el fundamento que permite la interrelación; para la preservación del mismo, se deben tener en cuenta varios factores, como la capacidad de carga, el papel de turista y la clasificación de los atractivos del altiplano, como montaña, laguna, rio, caverna, parque natural, parque temático, santuario de flora o fauna, entre otros.

La empresa es la base donde se estructura la red, que cuenta con las funciones de producción, de finanzas, de comercialización y de administración del talento humano y clasificada de acuerdo a sus actividades complementarios de alojamiento, de alimentación, de transporte, de operadores de atractivos y de comercio, especialmente, de suvenires.

El Estado como parte de la red, lo conforman las entidades gubernamentales como el Vice-ministerio de turismo y las secretarias departamentales y municipales 
que promueven el turismo y los organismos de control, cuyo apoyo sería solicitado por las empresas conformantes de la red.

El turista es el principal factor de la red de ecoturismo; la forma en que interactúe con el medio y su percepción determina su experiencia: el turista puede ser espectador, actor o actor-observador.
Se identificó que los componentes necesarios para la conformación de una red en el altiplano Cundiboyacense son los turistas, las empresas, la comunidad y el Estado, que interactúan en torno a los atractivos naturales y que responden cada uno a necesidades específicas, de acuerdo a los servicios ofrecidos. La red, se sintetiza en la figura 1.

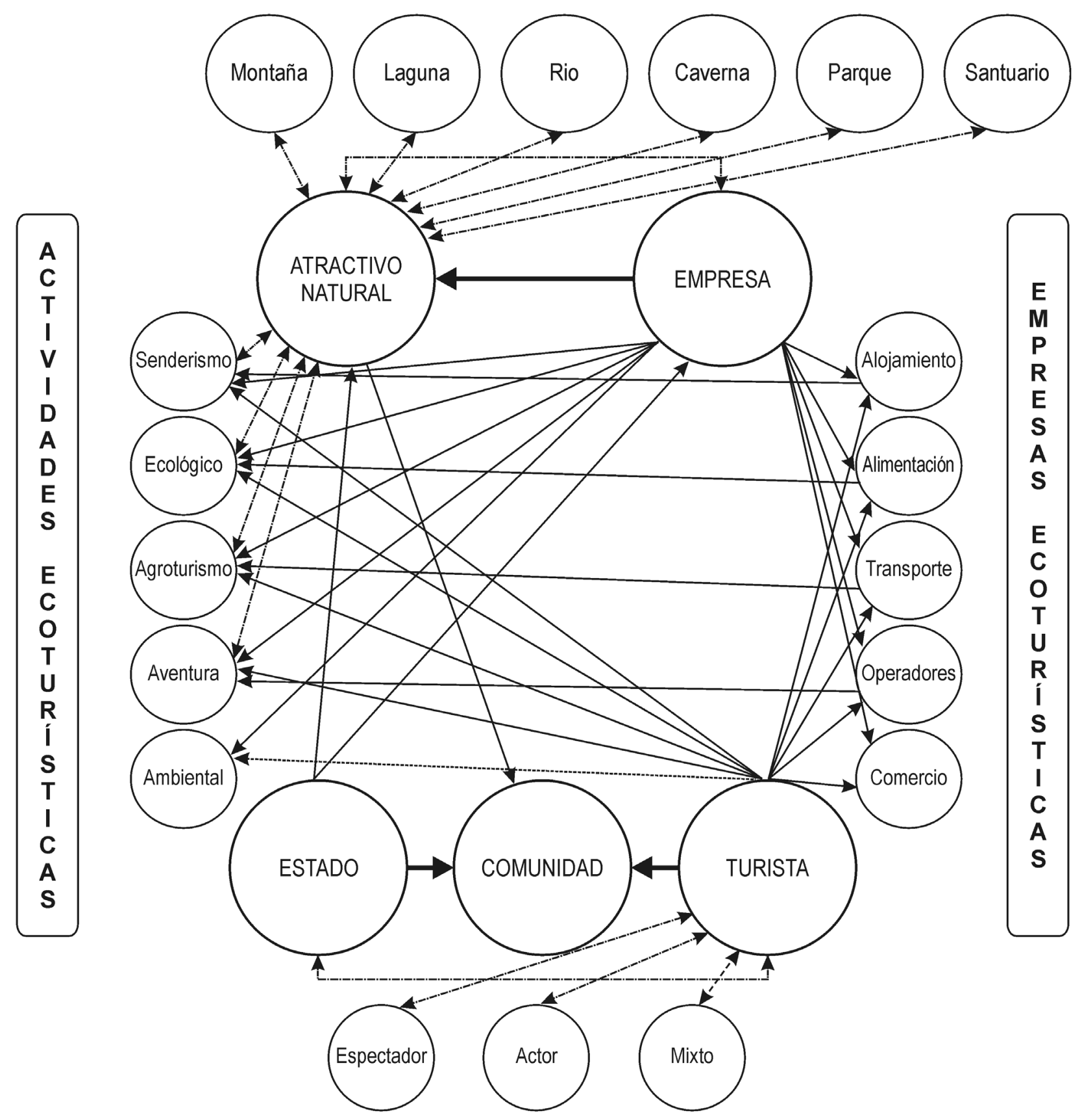

Figura 1. Propuesta de red para la actividad eco-turística. 
Es por esto, que se hace necesario sensibilizar a la comunidad del altiplano para que participe en la conformación de la red e, igualmente, proponer una estrategia de comunicaciones que de a conocer la región como un todo, que resalte las fortalezas de la integración y las actividades que se pueden realizar en los atractivos naturales, promoviendo los servicios complementarios, ofrecidos por las empresas de la red

Conflictos de intereses: El manuscrito fue preparado y revisado con la participación de todos los autores, quienes declaramos que no existe ningún conflicto de intereses que ponga en riesgo la validez de los resultados presentados. Financiación: Este estudio fue financiado por la Universidad de Ciencias Aplicadas y Ambientales U.D.C.A.

\section{BIBLIOGRAFÍA}

1. ANDREU, L.; PARRA, E. 2005. Gestión de Redes en Empresas y Destinos Turísticos. Turismo y Patrimonio Cultural (España). 5(3):399-402.

2. ANUARIO TURISMO Y SOCIEDAD, UNIVERSIDAD EXTERNADO DE COLOMBIA. 2005. Islas de Providencia y Santa Catalina, Presente y Futuro. La Institución (Bogotá). 185p.

3. BRENES, E.; PORRAS, M. 2004. Teoría de la Educación. Ed. Universidad Estatal a Distancia UNED. (España). 505p.

4. CAMARA, R.; MARTINEZ, B.J.; DÍAZ DEL OLMO, F. 2005. Desarrollo sostenible y medio ambiente en República Dominicana. Universidad de Sevilla. (España). 280p.

5. CEBALLOS, H. 1991. Tourism, eco-tourism and protected areas. In: Kusler, J.A. ed. Eco-tourism and Resource Conservation (Switzerland). 120p.

6. CENTRO DE ESTUDIOS AGROPECUARIOS. SERIE DE AGRO NEGOCIOS. 2001. Turismo rural y ecoturismo. Grupo Editorial Iberoamericana (México). 111p.

7. COLOMBIA. MINISTERIO DE AMBIENTE, VIVIENDA Y DESARROLLO TERRITORIAL.1996. Ley 300 de julio 26 de 1996. Por la cual se expide la Ley
General de Turismo y se dictan otras disposiciones. Diario Oficial No. 46.461 fecha publicación. 30 de julio de 2006. Bogotá, Colombia. Imprenta Nacional, 41p.

8. COLOMBIA. MINISTERIO DE AMBIENTE, VIVIENDA Y DESARROLLO TERRITORIAL 2006. Ley 1101 de 22 de noviembre de 2006 Por la cual se modifica la Ley 300 de 1996 - Ley General de Turismo y se dictan otras disposiciones. Diario Oficial No. 46.461 fecha publicación. 23 de noviembre de 2006. Bogotá, Colombia. Imprenta Nacional, 11p.

9. DÍAZ, J. 2001. Propuesta para el desarrollo de un producto ecoturístico en el sector 1 "Los Pinos" el Parque Nacional Natural Sumapaz. (Bogotá). Universidad Externado de Colombia. 156p.

10. FRED, D. 2008. Conceptos de administración estratégica. Pearson Educación (México). 416p.

11. FERNÁNDEZ, E. 2005. Estrategia de innovación. Thomson Ed. (España). 615p.

12. GALÁN, I. 2007. Diseño Organizativo. Thomson Ed. (España). 375p.

13. HABA, P. 2004. Elementos Básicos de Axiología General. Ed. Universidad de Costa Rica. (Costa Rica). 402p.

14. INSTITUTO ALEXANDER VON HUMBOLDT Y PONTIFICIA UNIVERSIDAD JAVERIANA - FACULTAD DE ESTUDIOS AMBIENTALES Y RURALES DEPARTAMENTO DE ECOLOGÍA Y TERRITORIO. 2004. Caracterización Biofísica del Altiplano Cundiboyacense (Bogotá). 456p.

15. INSTITUTO DE INVESTIGACIÓN DE RECURSOS BIOLÓGICOS ALEXANDER VON HUMBOLDT. 2000. Biocomercio: estrategias para el desarrollo sostenible en Colombia. Quiceno Mesa, M.P. (ed.). Instituto v. Humboldt (Bogotá). 271p.

16. KENT, M. 2003. Ecotourism, environmental preservation and conflicts over natural resources. Horizon Anthropological. 9(20):185-203.

17. LAGUNAS, D. 2008. Antropología y turismo: claves culturales y disciplinares. Ed. Plaza Valdes (México) 260p. 
18. LARA DE VICENTE, F. 2005. Turismo Sostenible, un enfoque Multidisciplinario. Universidad de Córdoba (España). 139p.

19. LOVELOCK, C.; WIRTZ, J. 2009. Marketing de servicios, personal, tecnología y estrategia. $6^{a}$ ed. Pearson Educación (México). 647p.

21. MUNÁRRIZ, L.; DOMÍNGUEZ, E. 2006. La conciencia humana Perspectiva cultural. Anthropos Editorial. (España). 303p.

22. MUÑ̃Z, M.A. 2005. Logística y turismo. Ed. Díaz de Santos. (España). 168p.

23. ORGANIZACIÓN MUNDIAL DE TURISMO OMT. 2002. OMT- PNUMA - Documento conceptual - Año internacional del ecoturismo. Disponible desde Internet en: www.cinu.org.mx/eventos/.../ doc_conceptual.htm (con acceso 15/02/10).
24. PÉREZ DE LAS HERAS, M. 2003. La Guía del Ecoturismo: o cómo conservar la naturaleza a través del turismo. Ed. Mundi-Prensa Libros. (España). 290p.

25. PALAFOX, A. 2005. Turismo: teoría y praxis. Plaza y Valdez Eds. (España). 338p.

26. PUIG, A. 2006. Los nuevos negocios turísticos. Valleta Ediciones, Argentina. 430p.

27. RHODES, A. R. 2005. Definiendo Ecoturismo. Disponible desde Internet en: www.ecoturismolatino. com (con acceso 30/11/09).

28. RIVANO, J. 2005. Lógica Elemental. $2^{\mathrm{a}}$ Ed. Ed. Universitaria el Saber y la Cultura. Chile. 221p.

29. SANDOVAL, S. 2007. Ecoturismo: operación técnica y gestión ambiental. Ed Eduforma (España), 237p.

Recibido: Noviembre 23 de 2009

Aceptado: Abril 11 de 2010 